\title{
CERTAIN FINITELY GENERATED COMPACT ZERO DIMENSIONAL SEMIGROUPS
}

\author{
R. P. HUNTER \\ (Received 28 January 1986; revised 23 January 1987) \\ Communicated by T. E. Hall
}

\begin{abstract}
Consider a compact zero dimensional (profinite) monoid. While the group of units must be open, a regular $D$-class need not be open in the ideal it generates. This is the case if and only if the semigroup contains infinitely many copies of a certain semilattice composed of an increasing sequence of idempotents converging to an upper bound.

Using compactifications of free products, two generator compact monoids with these properties are constructed.
\end{abstract}

1980 Mathematics subject classification (Amer. Math. Soc.): 22 A 15.

This note is in part a continuation of [2] and [5] where finitely generated compact zero dimensional semigroups were studied via the semigroup of continuous endomorphisms. (A compact semigroup is finitely generated if it contains a dense finitely generated (abstract) semigroup.)

In a compact finitely generated compact monoid, the group of units is both open and closed. This is easy to see and it would seem plausible that this property is local. (A property of a semigroup $S$ is called local if it is appropriately held by each subsemigroup of the form $e S e$ where $e$ is idempotent.) It is somewhat surprising that this is not the case. Questions concerning the openess of a maximal subgroup in the monoid it generates or of a $D$-class in the ideal it generates arise naturally in the factoring of homomorphisms into those of special type and in questions on the continuity of certain endomorphisms.

(C) 1988 Australian Mathematical Society $0263-6115 / 88 \$ A 2.00+0.00$ 
In this note we establish two results on the local structure of the semigroups considered. For completeness and because of its importance we present a self contained short proof of Numakura's result that any compact zero dimensional semigroup is profinite. This will be done through a simple application of the syntactic (or principal) congruence.

It will be convenient to have some notation: let $I_{0}$ denote the natural numbers with $\infty$ adjoined in the usual way with the multiplication $x y=\min (x, y)$. Thus $I_{0}$ is a simple sequence of idempotents $e_{1}, e_{2}, e_{3}, \ldots$ converging to $e$ with $e_{1}<e_{2}<e_{3}<\cdots$.

Let $T_{0}$ denote the usual Cantor set with the multiplication $x y=$ minimum $(x, y)$ and let $B$ denote $\{0,1\}$ with the same minimum multiplication.

If $S$ is finitely generated compact and zero dimensional then $S$ is the inverse limit of a sequence of finite semigroups $S_{1}, S_{2}, S_{3}, \ldots, S_{i}, \ldots$ where each $S_{i}$ is obtained from $S$ by a congruence which is invariant under the semigroup of continuous endomorphisms. Of course $S$ is, in particular, metric.

The principal results are contained in the following two theorems.

THEOREM A. Let $S$ be a finitely generated compact zero dimensional semigroup. Then $S$ contains a copy of $I_{0}$ if and only if some regular D-class outside of the minimal ideal fails to be open in the ideal it generates. If $S$ contains one copy of $I_{0}$ it contains an infinite collection of copies any two of which are isomorphic in such a way that corresponding elements are D equivalent.

THEOREM B. There exists a two generator profinite inverse monoid which contains copies of $I_{0}$ and $T_{0}$ and, in fact, contains a copy of $B^{\omega}$ the cartesian product of a countable number of copies of $B$.

THEOREM C (NUMAKURA [6]). A compact zero dimensional semigroup is the inverse limit of finite semigroups.

We begin with lemmas which may be of independent interest.

Let $S$ be a compact (or stable [1]) semigroup and let $D$ be a regular $D$-class. If $e^{2}=e \in D$ then $e S e \cap D=H_{e}=$ the maximal subgroup of $e([1])$. This immediately yields

LEMMA 0. If the compact semigroup $S$ contains a copy of $I_{0}$ then the maximal subgroup at $e$ is not open in eSe and the D-class at $e$ is not open in the ideal it generates.

LEMMA 1. Let $S$ be a metric profinite monoid whose group of units is not open. Then $I_{0}$ is imbedded as a submonoid. 
Proof. Let $V$ be any open set containing $G$ the group of units. Since $S$ is profinite there exists an open and closed submonoid $M$ with $V \supset M \supset G$. Since $G$ is not open the minimal ideal of $W$ is disjoint from $G$. Thus there is a descending sequence of submonoids $M_{1} \supset M_{2} \supset M_{3} \supset \cdots$ closing down on $G$. Let $K_{i}$ denote the minimal ideal of $M_{i}$. If $f_{i}$ is an idempotent of $K_{i}$ there exist idempotents $f_{j} \in K_{j}$ for $j<i$ such that $f_{j_{0}}<f_{j}$ if and only if $j_{0}<j$. Thus, for each $i=1,2,3, \ldots$ there is a chain of idempotents $e_{1}^{i}<e_{2}^{i}<e_{d}^{i}<\cdots<e_{i-1}^{i}<e_{i}^{i}$ where $e_{q}^{i} \in K_{q}$. Denote this chain of idempotents by $E^{i}$. Now the sequential limiting set $E^{\infty}$ of a subsequence of $\left\{E^{i}\right\}$ contains an idempotent $e_{i}$ from each $K_{i}$. Moreover $e_{1}<e_{2}<\cdots<e_{i}<e_{i+1}<\cdots$. Finally, the identity element 1 being the only idempotent in $G$ also belongs to $E^{\infty}$.

LEMMA 2. Let $S$ be a finitely generated profinite semigroup. Let $D$ be a $D$-class such that $D / H$ is finite. Then $D$ is open in $J(D)$.

Proof. Fix an $\not$-class of $D$, say $H$, and let $x_{1}, x_{2}, \ldots, x_{n}$ denote the generators. Let $T$ be the union of all translates $x_{i} H_{\alpha}$ and $H_{\alpha} x_{i}$, (where $H_{\alpha}$ runs through the $H$-classes of $D$ and $x_{i}$ is a generator), which are contained in the ideal $J(D) \backslash D$. Then $T$ is compact and $J(T)$ is a closed ideal contained in $J(D) \backslash D$.

Let $V$ be an open set about $H$ such that $V \cap J(T)=\varnothing$, and $V \cap D=H$. Suppose that $S H S \backslash H$ meets $V$ so that for some $s, t \in S$ one has $s H t \cap V \neq \varnothing$. Then for $w, w^{\prime}$, words in the generators, one has $w H w^{\prime} \cap V \neq \varnothing$. Let $w=$ $y_{1} y_{2} \cdots y_{k}$ and $w^{\prime}=y_{1}^{\prime} y_{2}^{\prime} \cdots y_{r}^{\prime}$ where the $y_{i}$ and $y_{j}^{\prime}$ are generators. Consider $y_{k} H y_{1}^{\prime}$. This is either an $\mathcal{H}$-class $H^{\prime}$ of $D$ or is contained in the ideal $J(T)$. If the latter, $w H w^{\prime} \subset J(T)$ and could not meet the open set $V$. If the former, look at $y_{k-1} H^{\prime} y_{2}^{\prime}$. Proceeding in this way, $w H w^{\prime}$ is either an $\mathcal{H}$-class of $D$ or is contained in $J(T)$. In either case one has a contradiction.

LEMMA 3. Let $S$ be a compact semigroup and $D_{e}$ a regular $D$-class of the idempotent $e$. If there are non idempotent $\mathcal{H}$-classes in $L_{e}$ arbitrarily close to $H_{e}$ then $H_{e}$ is not open in eSe.

PROOF. If $x_{\alpha} \rightarrow h \in H_{e}$ then $e x_{\alpha} e \rightarrow e h e=h$. Moreover if $x_{\alpha} \mathcal{L} e$ and $H_{x_{\alpha}}$ is not a subgroup it is known (see [1]) that $e x_{\alpha} \notin D_{e}$. Hence $e x_{\alpha} e$ is an element of $J(D) \backslash D$.

PROOF OF THEOREM A. Suppose $S$ is a finitely generated semigroup and $I_{0}$ is a subsemigroup of $S$. Consider $D_{e}$ the $D$-class of $e$ the identity of $I_{0}$. From Lemma 2 we know that $D_{e}$ has an infinite number of $\mathcal{H}$-classes and thus must contain an infinite number of $\mathcal{L}$-classes or an infinite number of $R$-classes. In either case, since each $\mathcal{L}$-class or $R$-class of $D_{e}$ must also contain an idempotent 
there are infinitely many idempotents in $D_{e}$. Finally if $f$ is another idempotent of $D_{e}$ then there is an isomorphism $x \mapsto a^{\prime} x a$ of $e S e$ onto $f S f$. Here $a \in R_{e} \cap L_{f}$ and $a^{\prime}$ be the inverse of $a$ in $R_{f} \cap L_{e}$. (See Theorem 2.18 of [3].) Thus, at each idempotent of $D_{e}$ there is a copy of $I_{0}$. Finally, the isomorphism is $D$-class preserving having as inverse $y \mapsto a y a^{\prime}$.

PROOF OF THEOREM B. For each prime we construct a certain finite semigroup $A_{p}$ : Let $C_{p}$ denote the cyclic group of order $p$ and let $B$ denote the two element monoid with $e$ as zero. Form the monoid $C_{p} * B$, the free product with the identities amalgamated. Let $J_{p}$ denote the ideal of $C_{p} * B$ generated by all words in which $e$ appears two or more times. Thus $J_{p}$ is generated by all elements of the form ege where $g \neq 1$. Let $A_{p}$ denote $C_{p} * B$ modulo the ideal $J_{p}$. Note that $A_{p}$ is a finite inverse monoid. With an obvious notation the group of units is $C_{p}$ and $C_{p} e C_{p}$ is a regular D-class with idempotents $g e g^{-1}, g \in C_{p}$.

Form the compact zero dimensional inverse monoid $\times A_{p_{i}}$ where $p_{i}$ runs over the primes. Within this monoid form $M=\operatorname{cl}\langle\tilde{g}, \tilde{e}\rangle$ the compact submonoid generated by $\tilde{g}=\left(g_{1}, g_{2}, g_{3}, \ldots\right)$ where $g_{i}$ generates $C_{p_{i}}$ and $\tilde{e}=(e, e, e, e, \ldots)$.

Since $\times C_{p}$ is a monothetic group it appears as $G$ the group of units in $M$. Note that the $\mathcal{L}$-class of $\tilde{e}$ is $G \tilde{e}$ which has only one idempotent. The existence of $I_{0}$ then follows from Lemma 2. To be explicit, let $\tilde{g}_{j}$ denote an element of $G$ such that the first $j$ coordinates are equal to 1 and all other coordinates are different from 1 . Then $\tilde{e} \tilde{g}_{j} \tilde{e}$ is a sequence of idempotents converging to $\tilde{e}$ from below. Thus, there is a copy of $I_{0}$.

However, let $\tilde{x}$ be an element with each coordinate equal to $e$ or 0 . Define the element $\tilde{y}$ by placing an $e$ in each coordinate where it appears as a coordinate of $\tilde{y}$ and placing $e g,(g \neq 1)$, in those coordinates where 0 is the coordinate of $\tilde{x}$. The element $\tilde{y}$ is clearly in $M$ and $\tilde{y} \tilde{e}=\tilde{x}$. Since $\tilde{x}$ is a typical element of $B^{\omega}$, the last must be a subsemigroup of $M$. The semigroup $B^{\omega}$ contains a copy of $T_{0}$ since there are sufficiently many continuous homomorphisms of $T_{0}$ into $B$ to separate points.

To see that $M$ is an inverse semigroup note first that the idempotents commute. One need only show that $M$ is regular. Any coordinate of a point of $M$ is of the form $0, e$ or $g e g_{0}$ where $g, g_{0} \in G$. Thus, if $\tilde{x} \in M$ the double orbit $G \tilde{x} G$ certainly contains an idempotent. This idempotent is certainly $D$ equivalent to $\tilde{x}$.

To prove the result of Numakura it suffices to show that a compact zero dimensional semigroup can be embedded, topologically and algebraically, as a subsemigroup of a cartesian product of finite semigroups. In effect, such a product is an inverse limit of the finite products. Moreover any compact subspace 
of an inverse limit is itself an inverse limit of its projections to the co-ordinate spaces.

Thus, it suffices to show that a compact zero dimensional semigroup has enough finite continuous homomorphisms to separate points, since this allows one to embed it into the Cartesian product of all the finite images. This will follow from Lemma 4 for which we recall the definition of $P_{A}$.

The syntactic (or principal) congruence is defined as follows: Let $A$ be any subset of a semigroup $S$. The points $x$ and $y$ of $S$ are congruent, $x P_{A} y$, if and only if

$$
u x v \in A \Leftrightarrow u y v \in A \text { for all } u, v \in S^{1} .
$$

(As usual $S^{1}$ denotes $S$ with an identity adjoined if necessary.)

It is clear that $P_{A}$ is needed a congruence in which $A$ is a union of classes.

The role of this congruence in compact zero dimensional semigroups can be seen from

LEMMA 4. Let $S$ be a compact semigroup and let $A$ be an open and closed subset of $S$. Then $P_{A}$, the syntactic congruence, is both open and closed.

Proof. To see that $P_{A}$ is closed suppose that $s$ and $t$ are inequivalent. Thus, there exist $u_{0}$ and $v_{0}$ such that, say, $u_{0} s v_{0} \in A$ while $u_{0} t v_{0} \notin A$. By continuity of multiplication, there exist open sets 0 about $s$ and $W$ about $t$ such that $u_{0} 0 v_{0} \subseteq A$ and $u_{0} W v_{0} \subseteq S \backslash A$. Then $0 \times W \subset(S \times S) \backslash P_{A}$.

To see that $P_{A}$ is open, we may suppose that $s_{\alpha} \rightarrow s, t_{\alpha} \rightarrow t$ with $s_{\alpha}$ and $t_{\alpha}$ inequivalent modulo $P_{A}$. There exist $u_{\alpha}$ and $v_{\alpha}$ such that $u_{\alpha} s_{\alpha} v_{\alpha} \in A$ and $u_{\alpha} t_{\alpha} v_{\alpha} \notin A$ (or vice versa). Since $S$ is compact, we may arrange things so that $u_{\alpha} \rightarrow u, v_{\alpha} \rightarrow v, s_{\alpha} \rightarrow s$ and $t_{\alpha} \rightarrow t$. Since $A$ is closed $u s v \in A$ and since $S \backslash A$ is closed $u t v \in S \backslash A$. But this says that $s$ and $t$ are inequivalent. Thus, the complement of $P_{A}$ is closed.

Finally, one notes that a compact semigroup modulo an open and closed congruence must be finite.

\section{References}

[1] L. W. Anderson, R. P. Hunter and R. J. Koch, 'Some results on stability in semigroups', Trans. Amer. Math. Soc. 17 (1965), 521-529.

[2] L. W. Anderson and R. P. Hunter, 'Homomorphisms having a given $H$-class as a single class', J. Austral. Math. Soc. 15 (1973), 7-14.

[3] A. H. Clifford and G. B. Preston, Algebraic theory of semigroups (Math. Surveys, no. 7, Amer. Math. Soc., 1961).

[4] K. H. Hofmann and P. S. Mostert, Elements of compact semigroups (Merrill, Columbus, Ohio, 1966). 
[5] R. P. Hunter, 'Some results on subgroups defined by the Bohr compactification', Semigroup Forum 26 (1983), 125-137.

[6] K. Numakura, 'Theorems on compact totally disconnected semigroups and lattices', Proc. Amer. Math. Soc. 8 (1957), 623-626.

\section{Department of Mathematics}

Pennsylvania State University

University Park, Pennsylvania 16802

U.S.A. 